\title{
Emergence of Toxicological Advancements: Historical Preview of
}

\section{$20^{\text {th }}$ Century}

\section{Tabassum Zafar* \\ Department of Biosciences, Barkatullah University, India}

*Corresponding author: Tabassum Zafar, Department of Biosciences, Barkatullah

University, B-40, NITTTR Campus, Shamla Hills, Madhya Pradesh, Bhopal-462026,

India, Email: tztabassumzafar@gmail.com

\section{Editorial \\ Volume 3 Issue 1}

Received Date: January 22, 2018

Published Date: February 01, 2018

\section{Editorial}

Toxicology is the overlapping branch of science, which extends dimensions within Chemistry, Biochemistry, Cell biology, Biotechnology, Pharmacology and Bioinformatics. Toxicology deals with study of toxic effects of chemicals on biological systems including plants, fishes, humans and animals. Now in $21^{\text {st }}$ century toxicology becomes one the popular and informative branch of science, which deals with diagnosis and predictions in very convenient and automated way. Now the developments in the field of toxicology are not limited to in vivo, in vitro, in silico testings and predictions but offer very fascinating outcomes. Biological interactions of toxicants via specific receptors, enzymes, or other discrete proteins and nucleic acids are now very easy to identify using modern techniques and procedures. Development of various new assays, detailed study of chemical interactions and advancement in drug delivery contribute significantly in better health management with each passing day. But during the rise of this scientific branch things were not as convenient as today. Like other branches of science toxicology also have a glorious past of evolution.

Toxicology as an identification tool has been successfully continued journey for more than sixty years. After the Second World War, emergence of new chemical moieties gave rise to specific concerns related to biological safety of those chemicals. During the period of 1930 to 1980 toxicological investigations of various toxicants were performed in relation to human health, wild life security and environmental toxicity. Investigating the cause of carcinogenesis also becomes a point of interests for researches. In the second half of $20^{\text {th }}$ century assessment of biochemical markers for hepatotoxicity and nephrotoxicity gains considerable popularity. Cell membrane and cell organnels also becomes a target of toxicological investigations. Many chemicals like 2,4-dinitrophenol, antineoplastic drugs (eg. vinca alkaloids) and neurotoxicants (eg. n-hexane), bromobenzene, acetoaminophene and many other chemicals were investigated for their potential toxic effects during the similar period. Uncoupling of oxidative phosphorylation was reported as the major pathway for all toxicological progressions. Involvement of glutathione in maintenance of homeostasis is also a remarkable investigation of the similar time period. In between 1960 to 1980 studies related to cytochrome P 450 comes in fusion with emergence of spectrophotometer as an important instrument. By the end of $20^{\text {th }}$ century it was unanimously accepted among researchers that depletion of glutathione is the remarkable cause of cellular toxicity, which results in tissue injury, cellular toxicity and genetic material damage. The interest of toxicologist then shifted towards the genetics of toxicity. During late $20^{\text {th }}$ century the focus of toxicological investigation shifted particularly on involvement of molecular diagnostic techniques in toxicological investigations along with the latest advancements of biotechnology. The consistent curiosity of toxicologists for molecular basis of toxicity enhances the developments of molecular tools for toxicity predictions and modulations. Molecular cloning, protein expression and biotransformation studies open new paradigms in front of researchers to explore molecular and biochemical pathways involved in progression of toxicity. Emergence of DNA amplification, transformation assays, xenobiotic testings, transcription factors, genes and protein super families contributes significantly for 


\section{Advances in Clinical Toxicology}

the progression of high throutput screening of toxicants. With latest advancements now it is even become possible to modify the conditions to restrict the level of toxicity in vitro as well as in vivo. Now a days bioinformatic approaches even allow predicting the toxicity levels of any chemicals before the synthesis of chemical. From tissue toxicity to cellular toxicity and then molecular toxicity the toxicology has extended dimensions continuously for the benefit of mankind. 